\section{THERAPEUTICS}

\section{Blade runner}

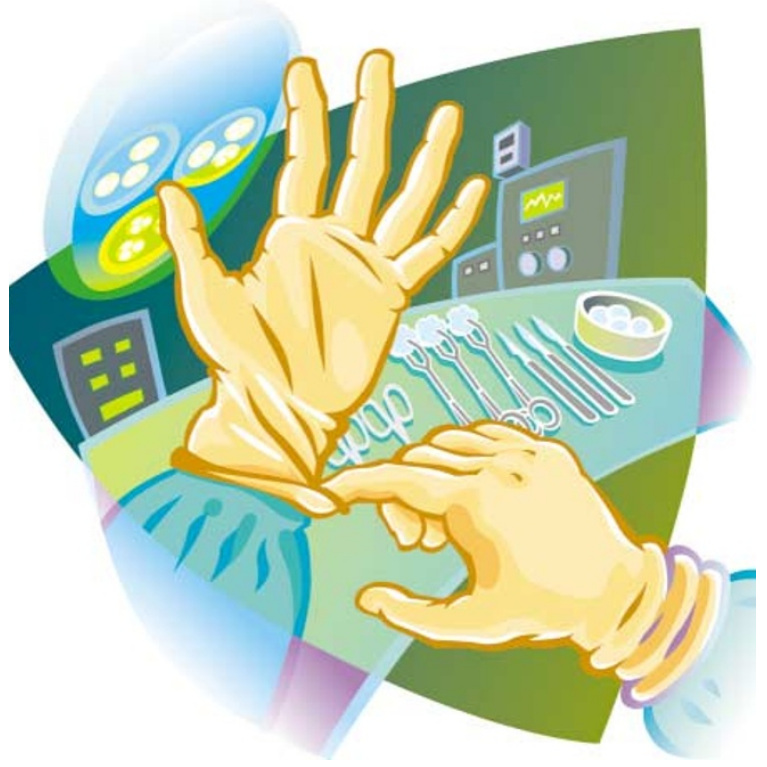

For patients with certain types of pituitary adenoma, surgery - often followed by radiotherapy - has been the only option. But now, Heaney et al. have identified the nuclear hormone receptor PPAR- $\gamma$ (peroxisome proliferator-activated receptor- $\gamma$ ) — which functions as a ligand-dependent transcription factor - as a potential therapeutic target that could provide an alternative to the surgeon's knife.

The most common pituitary tumours do not secrete hormones (non-functioning adenomas) and there are no effective drug therapies for these tumours. In addition, a subset of patients with prolactin (PRL)- and growthhormone $(\mathrm{GH})$-secreting tumours do not respond to the commonly used dopamine agonists or somatostatin analogues. Patients with both types of tumour require surgery. Most patients are diagnosed only when hormonal hypersecretory syndromes have become evident or when their tumours have reached more than $1 \mathrm{~cm}$ in diameter, and surgical resection of these macroadenomas has a cure rate of only $\sim 30 \%$. Surgery also risks damage to surrounding normal pituitary tissue, leading to hormonal deficiency (which is an important cause of co-morbidity).

Expression of PPAR- $\gamma$ is upregulated in prostate, breast and colorectal tumours, and treatment with PPAR- $\gamma$-activating thiazolidinediones (TZDs, such as rosiglitazone and troglitazone) inhibits the growth of such tumours in vitro and in mouse models. The authors of this study have now shown that PPAR- $\gamma$ is also highly expressed in human pituitary tumour tissue compared with normal pituitary tissue, and that TZDs inhibit growth of these tumours in vitro and in vivo.

Treatment of human non-functioning, rat GH3 (PRL- and GH-secreting) or mouse $\alpha$ T3 (gonadotroph) pituitary tumour cells with rosiglitazone or troglitazone led to G0-G1 cell-cycle arrest and decreased the number of cells in $\mathrm{S}$ phase. This was shown to be associated with a decrease in the level of phosphorylated retinoblastoma protein. Treatment with TZDs also increased tumour-cell apoptosis in vitro in a dose-dependent manner, which correlated with an increase in the level of the pro-apoptotic protein Bax.

\title{
Trojan horses
}

Tumours co-opt neighbouring blood vessels, along with vascular endothelial cells from those vessels, to support their own blood supply. They are also capable of attracting haematopoietic cells that contribute to the formation of their own blood vessels. In the June issue of Nature Medicine, De Palma et al. have shown that it is possible to use these cells as a 'Trojan horse' to deliver suicide genes to the developing tumour.

Tumours produce growth factors that induce bone-marrow-derived endothelial progenitor cells to migrate to the tumour, where they contribute to the developing vasculature. To track these cells, DePalma et al. created lentiviral vectors that express green fluorescent protein (GFP) from promoter and enhancer sequences of the Tek gene, which encodes a receptor tyrosine kinase that is expressed almost exclusively in endothelial cells. The authors transduced a variety of cell types with the vector, and showed that GFP was expressed specifically in endothelial cells.

Mouse bone-marrow cells were transduced with the vector and transplanted back into their hosts. Within 12-24 weeks of transplantation, GFP was expressed by a small fraction of bone-marrow cells. Tumours were then grafted into these mice, and the GFP-expressing cells localized to the periphery of melanomas and other types of carcinomas. Here, they were closely associated with non-labelled endothelial cells in cord-like, laminar structures. There were fewer GFP-positive cells found in the inner tumour mass, but those present were often associated with small blood vessels. De Palma et al. could not find evidence that the transduced cells developed into bona fide vascular endothelial cells. Rather, the cells expressed monocyte lineage markers and seemed to represent a subset of endothelial progenitors that promote angiogenesis by an unknown mechanism.

So, can these cells be used to deliver celldeath-inducing genes to tumours? DePalma et al. developed a 'suicide' vector containing a Tek promoter-driven thymidine kinase gene, which kills cells when expressed in the presence of gancyclovir. Bone-marrow cells were transduced with this suicide vector, transplanted back into mice and tested for their effects on tumour development. The

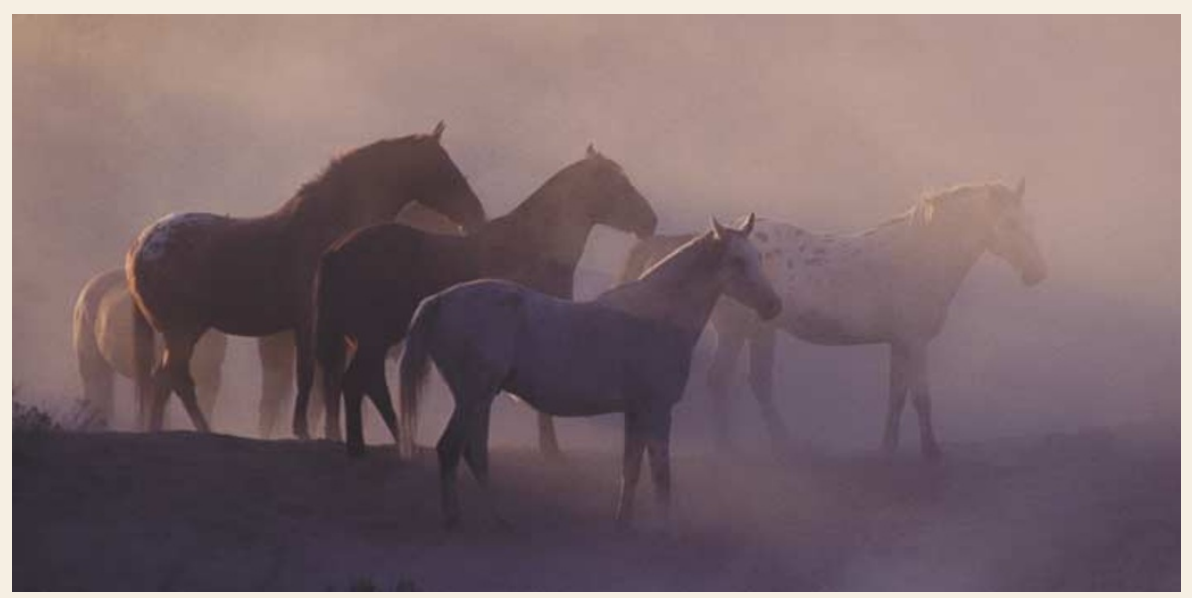


In an in vivo model, treatment with rosiglitazone was shown to inhibit tumour growth in athymic nude mice that were inoculated subcutaneously with GH3 cells tumour weight was markedly lower in treated mice compared with control mice after 4 weeks. Importantly, rosiglitazone could also inhibit the growth of already established tumours of $\alpha \mathrm{T} 3$ cells.

As oral rosiglitazone has already been approved for use in humans in the United States (for the treatment of type-2 diabetes), this drug could be safer and more effective than going under the knife for patients with non-functioning adenomas or with hormone-secreting pituitary tumours that are unresponsive to current drug therapies.

Kirsty Minton

(2) References and links

ORIGINAL RESEARCH PAPER Heaney, A. P., Fernando, M. \& Melmed, S. PPAR- $\gamma$ receptor ligands: novel therapy for pituitary adenomas. J. Clin. Invest. 111, 1381-1388 (2003) FURTHER READING Heaney, A. P., Fernando, M., Young, W. \& Melmed, S. Functional PPAR- $\gamma$ receptor represents a novel therapeutic target in Cushing's disease. Nature Med. 11, 1281-1287 (2002)

vector-expressing cells again localized to the tumours, but on exposure to gancyclovir, were quickly cleared. At the end of the treatment phase, these tumours were much smaller and their vascular density was almost fivefold less than control tumours. There were no signs of myelotoxicity in treated mice.

DePalma et al. also checked to see if the transduced haematopoietic cells homed to other sites of neo-angiogenesis, such as to healing wounds. They found that after partial hepatectomy, Tek/GFP-expressing cells localized to the granulation tissue that surrounds regenerating hepatic lobules. This cancer treatment might therefore disrupt wound-healing in patients.

The authors conclude, however, that haematopoietic cells are a useful vehicle for delivering gene-based therapies to tumours. The bone-marrow-derived cells that associate with the developing tumour vasculature will have to be better characterized, but other reagents designed to target these cells might be developed as antitumour agents.

Kristine Novak

(9) References and links ORIGINAL RESEARCH PAPER DePalma, M., Venneri, M. A. \& Naldini, L. Targeting exogenous genes to tumo angiogenesis by transplantation of genetically modified hematopoietic stem cells. Nature Med. 9, 789-795 (2003) FURTHER READING Rafii, S. et al. Vascular and haematopoietic stem cells: novel targets for antiangiogenesis therapy. Nature Rev Cancer 2 826-835 (2003) WEB SITE

Luigi Naldini's lab:

http://lgxserver.uniba.it/lei/rassegna/naldini.htm

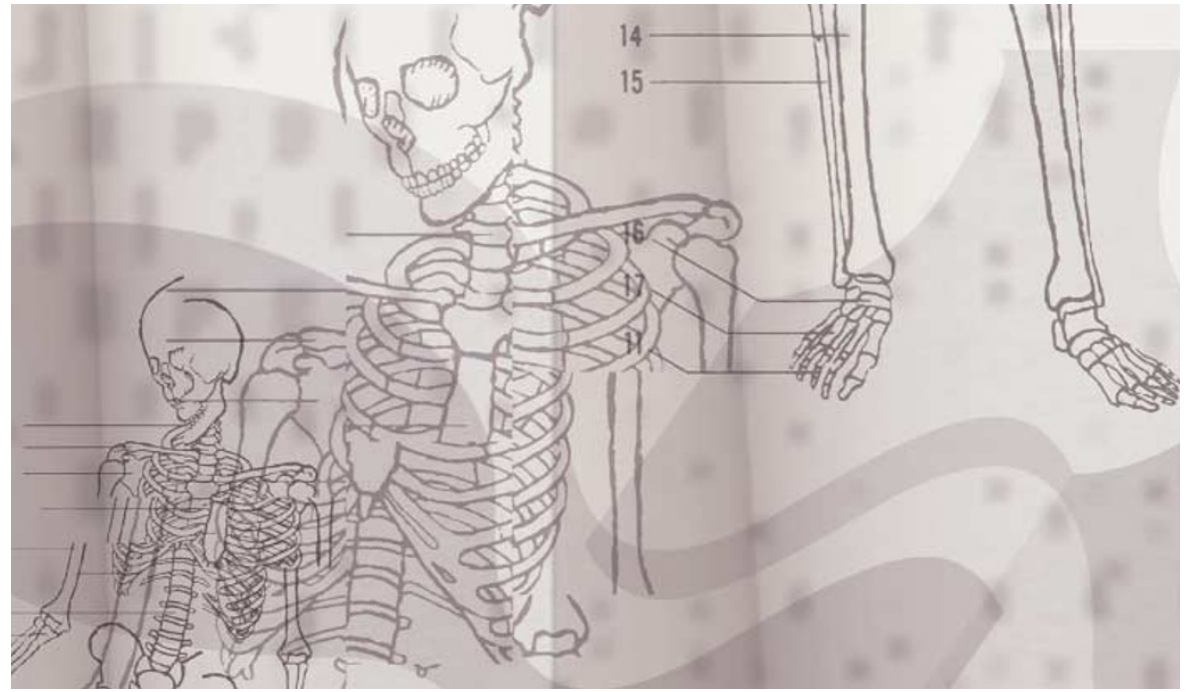

ANGIOGENESIS

\section{Skeletal links}

When tumours become large and hypoxic, cancer cells activate hypoxia-inducible factor (HIF), leading to transcription of vascular endothelial growth factor (VEGF) and other factors that promote angiogenesis. Mabjeesh et al. now show that the antitumour agent 2-methoxyestradiol (2ME2) - known to have anti-angiogenic properties - dysregulates HIF and that this function is linked to disruption of the microtubule cytoskeleton.

$2 \mathrm{ME} 2$ is a naturally occurring derivative of oestradiol and preliminary results from Phase I and II trials in patients with breast and prostate cancer have shown promising antitumour responses. In investigating its effects in human prostate cancer and breast cancer cell lines, the authors observed that 2ME2 decreased the levels of the alpha subunit of HIF1 (HIF-1 $\alpha$ ), leading to reduced expression of HIF- $1 \alpha$ target genes, including VEGF. Exposure of cells to cycloheximide - which blocks new protein synthesis - caused a similar rate of decrease in HIF- $1 \alpha$ levels in both 2ME2treated and -untreated cells, indicating that 2ME2 inhibits synthesis but not stability of HIF-1 $\alpha$. To examine the effect on protein translation further, the authors carried out ${ }^{35} \mathrm{~S}$-methionine labelling experiments and showed that HIF-1 $\alpha$ synthesis was higher in untreated cells compared with 2ME2treated cells 15 minutes after labelling. In addition, use of a proteasome inhibitor led to an increase of HIF- $1 \alpha$ levels in untreated cells but did not restore the inhibitory effect of $2 \mathrm{ME} 2$ on HIF1. Together, these data indicate that $2 \mathrm{ME} 2$ probably inhibits HIF- $1 \alpha$ protein synthesis rather than enhancing its degradation.

It has previously been shown that $2 \mathrm{ME} 2$ binds to tubulin and depolymerizes microtubules in both endothelial and tumour cells in vitro. So, is there a link between the effects of $2 \mathrm{ME} 2$ on the cytoskeleton and its effects on HIF- $1 \alpha$ ? Under hypoxia, cells treated with $2 \mathrm{ME} 2$ showed dose-dependent depolymerization of microtubules, and this disruption was necessary before a significant decrease in accumulation of HIF- $1 \alpha$ in the nucleus was seen compared with untreated cells.

To study the effect of the drug's activity on tumour microvascular density, mice bearing orthopically growing breast tumours were treated with doses of $2 \mathrm{ME} 2$ that are known to be efficacious in vivo. A dose-dependent reduction in tumour volume and a clear decrease in microvessels was seen with drug treatment. A laser-scanning confocal microscopy technique using an antibody against tubulin revealed that the microtubules of the treated tumour cells in the mice were depolymerized in a dosedependent manner and aberrant mitotic spindles were seen in nearly every cell. In addition, disruption of microtubules also occurred in endothelial cells of vessels associated with the tumours.

So, at concentrations that inhibit tumour growth and vascularization in vivo, $2 \mathrm{ME} 2$ effectively depolymerizes tumour microtubules. The authors also showed that other microtubule-targeting antitumour agents — vincristine and paclitaxel — inhibit HIF- $1 \alpha$, further supporting evidence of a link between disruption of the microtubule cytoskeleton and repression of HIF- $1 \alpha$, leading to downregulation of VEGF and inhibition of angiogenesis.

Ezzie Hutchinson

6) References and links

ORIGINAL RESEARCH PAPER Mabjeesh, N. J. et al. 2ME2 inhibits tumor growth and angiogenesis by disrupting microtubules and dysregulating HIF. Cancer Cell 3, 363-375 (2003)

WEB SITE

Paraskevi Giannakakou's lab:

www.winshipcancerinstitute.org/research/giannakakou_lab/ index.htm 